\title{
Facile Synthesis and Characterization of Au Nanoclusters-Silica Fluorescent Composite Nanospheres
}

\author{
Huiping Wang, Chaoyong Xu, Chengzhi Zheng, Wei Xu, Tianjiao Dong, Kanglei Liu, \\ Heyou Han, and Jiangong Liang
}

State Key Laboratory of Agricultural Microbiology, College of Science, Institute of Chemical Biology, Huazhong Agricultural University, Wuhan 430070, China

Correspondence should be addressed to Jiangong Liang; liangjg@mail.hzau.edu.cn

Received 13 June 2013; Revised 6 September 2013; Accepted 9 September 2013

Academic Editor: Lavinia Balan

Copyright (c) 2013 Huiping Wang et al. This is an open access article distributed under the Creative Commons Attribution License, which permits unrestricted use, distribution, and reproduction in any medium, provided the original work is properly cited.

\begin{abstract}
We developed a novel method for the synthesis of Au nanoclusters (NCs) silica fluorescent composite nanospheres by mixing the as-prepared bovine serum albumin (BSA) protected Au NCs with amino-modified silica spheres in acetate buffer solution. The products were characterized by high-resolution transmission electron microscopy (HRTEM), X-ray photoelectron spectroscopy (XPS), fluorescent microscope imaging (FLMI), and dynamic light scattering (DLS) measurements. The proposed method was simple, efficient, and inexpensive. In addition, the composite nanospheres exhibited favorable water-dispersible, stable, and fluorescent properties, potentially leading to further applications in chemical and biological sensors. A reasonable mechanism was also proposed for the formation of composite nanospheres.
\end{abstract}

\section{Introduction}

Recently, strong attention has focused on the synthesis of fluorescent composite nanospheres, such as quantum dots, dyes, and carbon dots-based composite materials, which are widely used as fluorescent probes in bioassay, sensing, and fluorescence imaging [1-4]. However, the information on fluorescent Au nanoclusters (NCs) based composite nanospheres is rather limited despite their unique electronic and optical properties. As an emerging fluorescent material, these fluorescent Au NCs possess a variety of distinct features, such as ultrasmall size $(<2 \mathrm{~nm})$, low toxicity, and lack of intermittency $[5,6]$. Therefore, Au NCs-based composite nanospheres can be potentially exploited for the development of new sensor application as well as fluorescent probes for in vivo and in vitro imaging. Le Guével et al. [7] reported the successful synthesis of fluorescent Au NCs-doped silica nanoparticles using a modified-Stöber method and subsequently used this new label for fluorescence imaging in tumor lung cells. Zhang et al. [8] developed a straightforward strategy for the preparation of fluorescent Au NCs with silica coating outer shells. Additionally, the application of these bioconjugated $\mathrm{Au}$ NCs-silica nanocomposites as bioprobes for cellular imaging was demonstrated. Samanta et al. [9] reported the synthesis of ultrasmall Au NCs arrays encapsulated in silica nanospheres and demonstrated the utilization of the composite material in fluorescence imaging and catalysis. Consequently, it is of great essential to explore more approaches for the preparation of Au NCs-based composite nanospheres.

In this work, we developed a simple method for the preparation of Au NCs-silica composite nanospheres. The morphology and optical properties of these as-prepared composite nanospheres have been investigated by HRTEM, XPS, FLMI, and DLS measurements. A possible formation mechanism of the composite nanospheres was also proposed.

\section{Experimental}

The BSA coated Au NCs were synthesized in one pot following the method developed by Xie et al. [10]. Briefly, $5.0 \mathrm{~mL}$ of $10 \mathrm{mM} \mathrm{HAuCl}_{4}$ solution was added to $5.0 \mathrm{~mL}$ of $50 \mathrm{mg} \cdot \mathrm{mL}^{-1}$ $\mathrm{BSA}$ solution under severe stirring at $37^{\circ} \mathrm{C}$. Two minutes later, $0.5 \mathrm{~mL}$ of $1.0 \mathrm{M}$ solution was added into it. The mixture was reacted at $37^{\circ} \mathrm{C}$ for $24 \mathrm{~h}$. The Au NCs were obtained when the color of the solution changed to deep brown, which was 


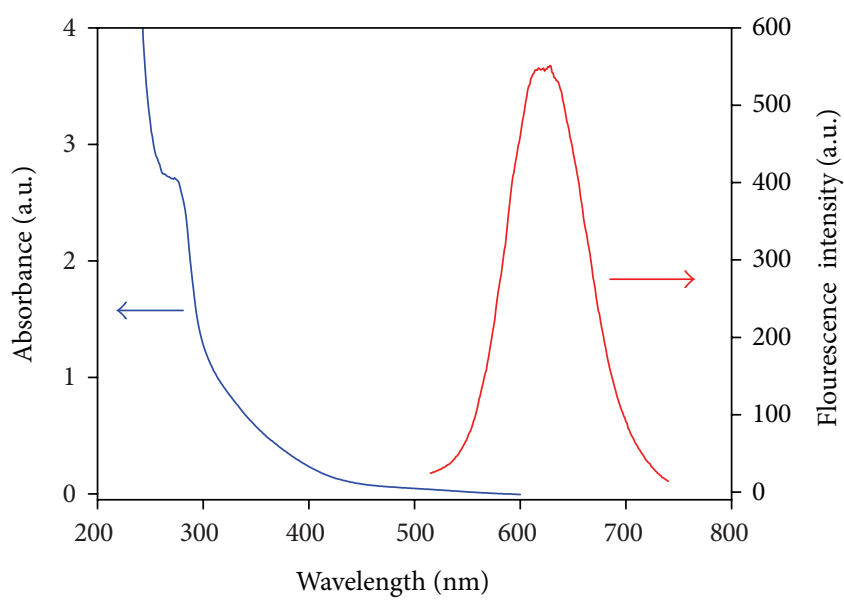

(a)

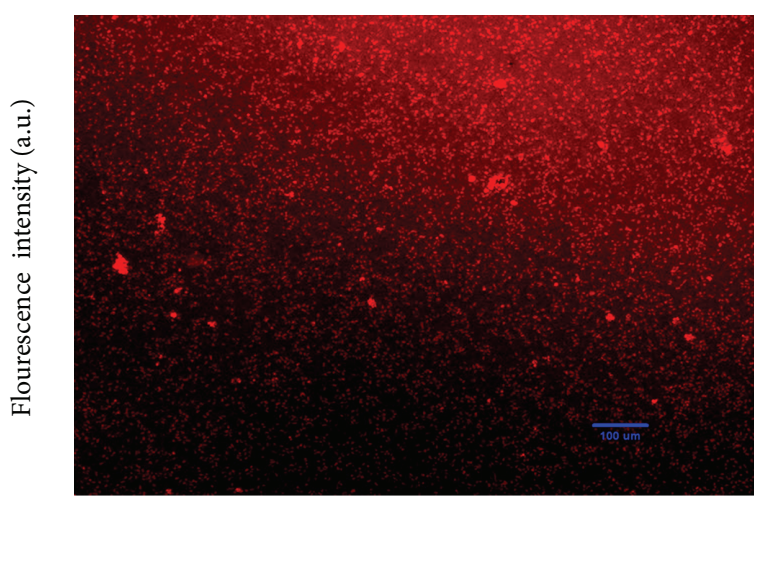

(b)

FIgURE 1: (a) Absorption and fluorescence emission spectra of BSA-Au NCs and (b) fluorescence microscope image of Au NCs-silica composite nanospheres (the scale bar is $100 \mu \mathrm{m}$ ).

dialyzed in membrane tubing with a molecular weight cutoff (MWCO) of $12 \mathrm{kDa}$ against $500 \mathrm{~mL}$ of ultrapure water under continuous stirring at room temperature to remove unreacted $\mathrm{HAuCl}_{4}$ or $\mathrm{NaOH}$.

Silica nanospheres were synthesized based on the Stöber method [11] and then were amino-functionalized by (3-aminopropyl)-trimethoxysilane. The as-prepared silica nanospheres were dispersed in sodium acetate-acetic acid buffer solution $(0.01 \mathrm{M}, \mathrm{pH}=5.0)$ under sonication. The purified $\mathrm{Au}$ NCs were added and mixed for 30 minutes. Then, the mixture was centrifuged and washed 3 times with ultrapure water. Finally, the composite nanospheres were resuspended in the buffer solution.

The UV-vis absorption spectrum was obtained in the range of $220-600 \mathrm{~nm}$, with $1.0 \mathrm{~cm} \times 1.0 \mathrm{~cm}$ quartz cuvette on an evolution $300 \mathrm{UV}$-vis spectrometer (Thermo Nicolet Corporation, USA). Fluorescence measurement was performed on a RF-5301PC Spectrofluorometer (Shimadzu, Japan) equipped with a $20 \mathrm{~kW}$ xenon discharge lamp as a light source. Fluorescence microscope image was recorded with a Ti-S fluorescence microscope (Nikon, Japan) with an objective (10x). The size and morphology of the products were acquired by a Tecnai G20 transmission electron microscope (FEI, USA) with an acceleration voltage of $200 \mathrm{kV}$. X-ray photoelectron spectroscopy (XPS) measurement was carried out by a VG Multilab 2000 spectrometer (Thermo Electron Corporation, USA) with an $\mathrm{Mg} \mathrm{K}_{\alpha} \mathrm{X}$-ray source. Hydrodynamic diameters and zeta potential of the samples were measured using a Zetasizer Nano ZS90 DLS system (Malvern, $\mathrm{UK})$.

\section{Results and Discussion}

The absorption spectrum in Figure 1(a) (blue line) exhibited a peak centered at $280 \mathrm{~nm}$, which was attributed to BSA molecules for stabilizing Au NCs [12]. Figure 1(a) (red line) showed the fluorescence emission spectrum of $\mathrm{Au}$ NCs with a peak around $628 \mathrm{~nm}$ upon excitation at $500 \mathrm{~nm}$.
Referred to previous reports, the emission was assigned to the intraband transitions of free electrons of Au NCs [13]. Figure S1 (see supplementary material available online at http://dx.doi.org/10.1155/2013/972834) indicated the fluorescence emission spectrum of Au NCs (black line) and the composite nanospheres (red line) upon excitation at $500 \mathrm{~nm}$. A small blue shift was observed, which may arise from the reduction of polarity in the local environment of $\mathrm{BSA}-\mathrm{Au}_{25}$ after attaching to the surface of silica nanospheres [14]. Figure S2 depicted the effect of illumination time with UV light on the fluorescence intensity of Au NCs (red line) and the $\mathrm{Au}$ NCs-silica composite nanospheres (black line). It can be seen that $\mathrm{Au}$ NCs-silica composite nanospheres have better stability than BSA/Au NCs. The fluorescence microscope image of the Au NCs-silica composite nanospheres was shown in Figure 1(b). These composite nanospheres were remarkably bright with red emission. It can be inferred that $\mathrm{Au}$ NCs were successfully attached to the surface of silica nanospheres, which led to the visible fluorescence of the composite material.

TEM images of silica nanospheres and fluorescent composite nanospheres were presented in Figure 2. The shape of silica nanospheres (Figure 2(a)) remained spherical, and the size was about $250 \mathrm{~nm}$. Compared with silica spheres, fluorescent composite nanospheres (Figure 2(b)) displayed some substance sticking to the surface, indicating the adhesion of BSA-Au NCs with silica nanospheres. Furthermore, some black spots could be seen on the composite nanospheres (Figure 2(b) inset), and these black spots $(\sim 1 \mathrm{~nm})$ were $\mathrm{Au}$ NCs on the nanosphere surface.

Binding properties and oxidation state of the powder sample of the composite spheres were confirmed by XPS (Figure 3). Au $4 \mathrm{f}_{7 / 2}$ for BSA-Au NCs could be deconvoluted into two distinct components centered at 84.1 (blue curve) and $85.0 \mathrm{eV}$ (red curve), which are ascribed to $\mathrm{Au}(0)$ and $\mathrm{Au}(\mathrm{I})$, respectively $[15,16]$, inferring that elemental $\mathrm{Au}$ NCs are entrapped in the composite spheres, which is in agreement with the TEM results. 


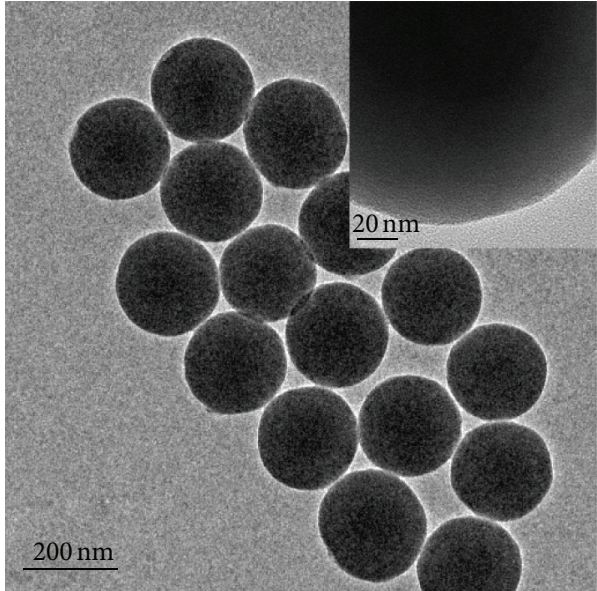

(a)

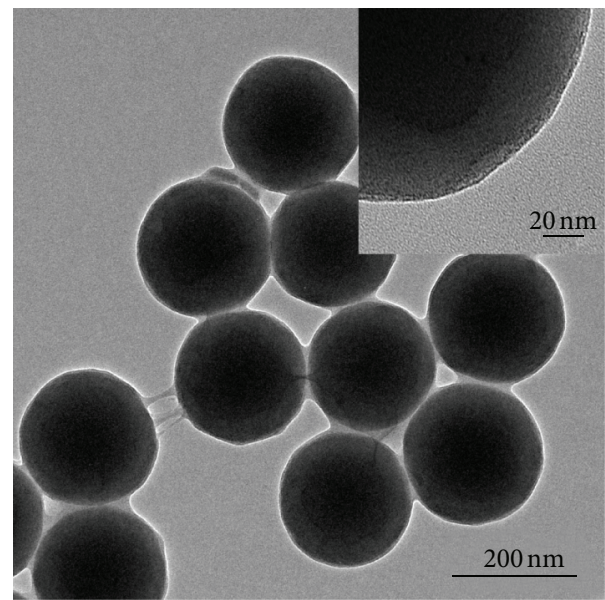

(b)

FIGURE 2: TEM images of (a) silica nanospheres and (b) Au NCs-silica composite nanospheres. The insets are the HRTEM images of a part of (a) silica nanospheres and (b) Au NCs-silica composite nanospheres.

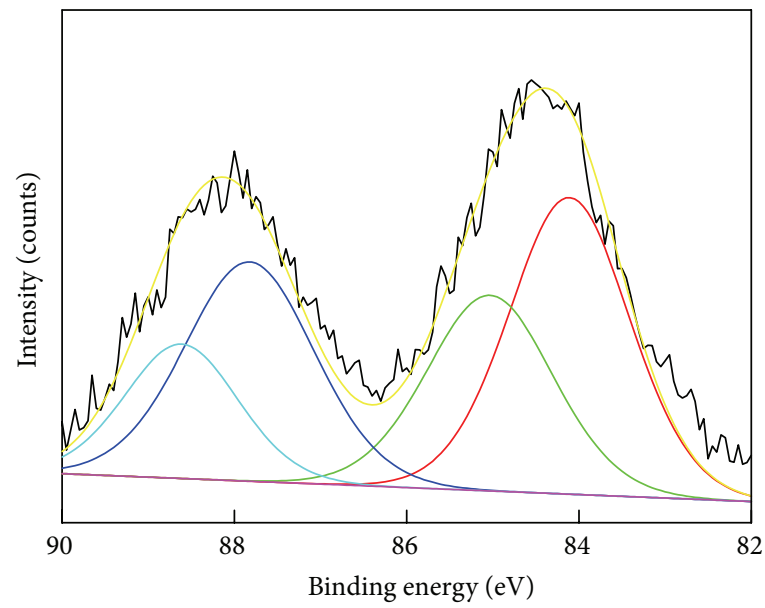

FIGURE 3: XPS spectra of Au 4f for Au NCs-silica composite nanospheres.

DLS has been used to measure the sizes of Au NCs, silica nanospheres, and the composite nanospheres. As shown in Figure 4, the hydrodynamic diameter of BSA-Au NCs and silica nanospheres was $10 \mathrm{~nm}$ and $257 \mathrm{~nm}$, respectively. However, the composite material showed a diameter of $3000 \mathrm{~nm}$, which was due to the adhesion of silica spheres together via BSA-Au NCs. The result here is coincident with the TEM image (Figure 2(b)).

Based on the above results, a possible formation mechanism of $\mathrm{Au} \mathrm{NCs}$-silica fluorescent composite nanospheres was suggested as follows (Figure 5). The zeta potential of the as-prepared BSA-Au NCs and silica nanospheres was determined to be $-13.7 \mathrm{mV}$ and $47.2 \mathrm{mV}$ at $\mathrm{pH} 5.0$, respectively. Therefore, these two materials were oppositely charged, suggesting that BSA-Au NCs and silica spheres could be conjugated by electrostatic interaction.

\section{Conclusion}

Herein, we described a straightforward method for the preparation of Au NCs-silica fluorescent composite nanospheres. The composite material exhibited favorable water solubility, stability, and strong fluorescence. An electrostatic interaction mechanism was proposed for the formation of composite nanospheres according to the negative charge of BSA-Au NCs and positive charge of amino-functionalized silica spheres in acetate buffer solution. The as-prepared composite material could be potentially applied to chemical and biological sensors.

\section{Authors' Contribution}

Huiping Wang and Chaoyong Xu contributed equally. 


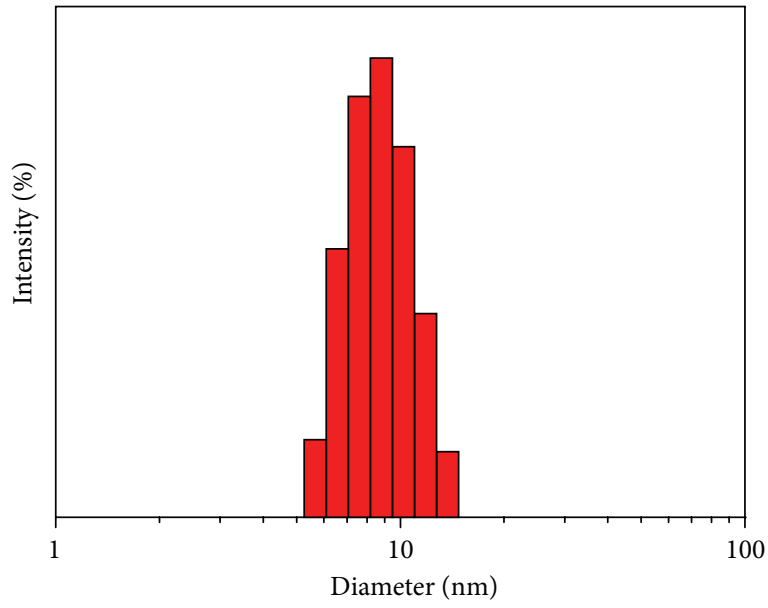

(a)

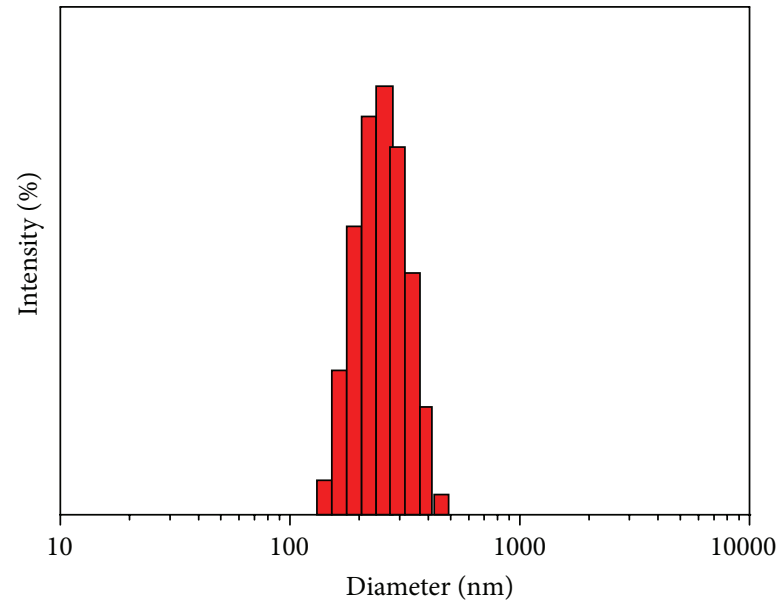

(b)

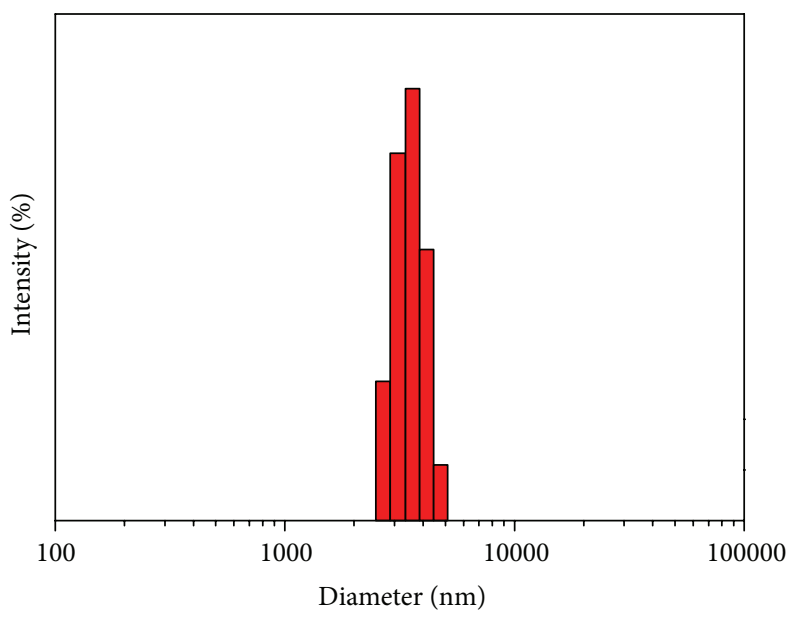

(c)

FIGURE 4: Size distribution histogram of (a) Au NCs, (b) silica nanospheres, and (c) Au NCs-silica composite nanospheres from the DLS measurement.

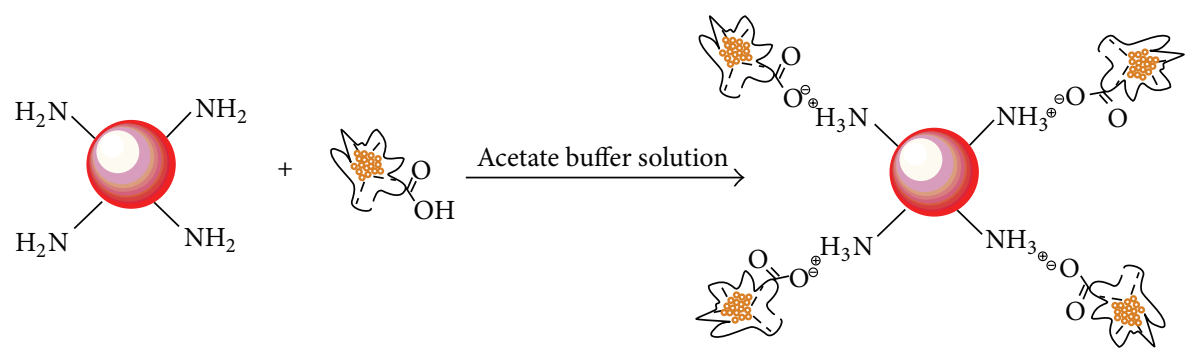

$$
\begin{array}{ll} 
& \text { Au NCs } \\
& \text { Silica spheres } \\
& \text { BSA }
\end{array}
$$

FIGURE 5: Schematic illustrations of formation mechanism of the Au NCs-silica composite nanospheres. 


\section{Acknowledgments}

This work was supported by Grants from National Natural Science Foundation of China (20905028), the Fundamental Research Funds for the Central Universities (2011PY009), Natural Science Foundation of Hubei Province Innovation Team (2011CDA115), and Huazhong Agricultural University Scientific \& Technological Self-innovation Foundation (2010SC05).

\section{References}

[1] Y. Zhao, Y. Ma, H. Li, and L. Wang, “Composite QDs@MIP nanospheres for specific recognition and direct fluorescent quantification of pesticides in aqueous media," Analytical Chemistry, vol. 84, no. 1, pp. 386-395, 2012.

[2] Y. L. Li, J. Zhou, C. L. Liu, and H. B. Li, "Composite quantum dots detect Cd(II) in living cells in a fluorescence "turning on" mode," Journal of Materials Chemistry, vol. 22, no. 6, pp. 25072511, 2012.

[3] X. Wang, S. Xu, and W. Xu, "Luminescent properties of dyePMMA composite nanospheres," Physical Chemistry Chemical Physics, vol. 13, no. 4, pp. 1560-1567, 2011.

[4] Y. Mao, Y. Bao, D. X. Han, F. H. Li, and L. Niu, "Efficient one-pot synthesis of molecularly imprinted silica nanospheres embedded carbon dots for fluorescent dopamine optosensing," Biosensors and Bioelectronics, vol. 38, no. 1, pp. 55-60, 2012.

[5] L. Shang, S. Dong, and G. U. Nienhaus, "Ultra-small fluorescent metal nanoclusters: synthesis and biological applications," Nano Today, vol. 6, no. 4, pp. 401-418, 2011.

[6] L. Shang and G. U. Nienhaus, "Gold nanoclusters as novel optical probes for in vitro and in vivo fluorescence imaging," Biophysical Reviews, vol. 4, no. 4, pp. 313-322, 2012.

[7] X. Le Guével, B. Hötzer, G. Jung, and M. Schneider, "NIRemitting fluorescent gold nanoclusters doped in silica nanoparticles," Journal of Materials Chemistry, vol. 21, no. 9, pp. 29742981, 2011.

[8] Z. Zhang, P. Zhang, K. Guo et al., "Facile synthesis of fluorescent $\mathrm{Au} @ \mathrm{SiO}_{2}$ nanocomposites for application in cellular imaging," Talanta, vol. 85, no. 5, pp. 2695-2699, 2011.

[9] A. Samanta, B. B. Dhar, and R. N. Devi, "Ultrasmall gold cluster arrays encapsulated in silica nanospheres: applications in fluorescence imaging and catalysis," Journal of Physical Chemistry C, vol. 116, no. 2, pp. 1748-1754, 2012.

[10] J. Xie, Y. Zheng, and J. Y. Ying, "Protein-directed synthesis of highly fluorescent gold nanoclusters," Journal of the American Chemical Society, vol. 131, no. 3, pp. 888-889, 2009.

[11] W. Stöber, A. Fink, and E. Bohn, "Controlled growth of monodisperse silica spheres in the micron size range," Journal of Colloid and Interface Science, vol. 26, no. 1, pp. 62-69, 1968.

[12] V. Poderys, M. Matulionyte, A. Selskis, and R. Rotomskis, "Interaction of water-soluble CdTe quantum dots with bovine serum albumin," Nanoscale Research Letters, vol. 6, no. 1, pp. 914, 2011.

[13] J. Zheng, P. R. Nicovich, and R. M. Dickson, "Highly fluorescent noble-metal quantum dots," Annual Review of Physical Chemistry, vol. 58, pp. 409-431, 2007.

[14] P. Yu, X. Wen, Y. Toh, Y. Lee, and J. Tang, "Optical properties of gold particle-cluster core-satellite nanoassemblies," RSC Advances, 2013.
[15] X. Le Guével, B. Hötzer, G. Jung, K. Hollemeyer, V. Trouillet, and M. Schneider, "Formation of fluorescent metal $(\mathrm{Au}, \mathrm{Ag})$ nanoclusters capped in bovine serum albumin followed by fluorescence and spectroscopy," Journal of Physical Chemistry C, vol. 115, no. 22, pp. 10955-10963, 2011.

[16] Y. Negishi, K. Nobusada, and T. Tsukuda, "Glutathione-protected gold clusters revisited: bridging the gap between gold(I)thiolate complexes and thiolate-protected gold nanocrystals," Journal of the American Chemical Society, vol. 127, no. 14, pp. 5261-5270, 2005. 

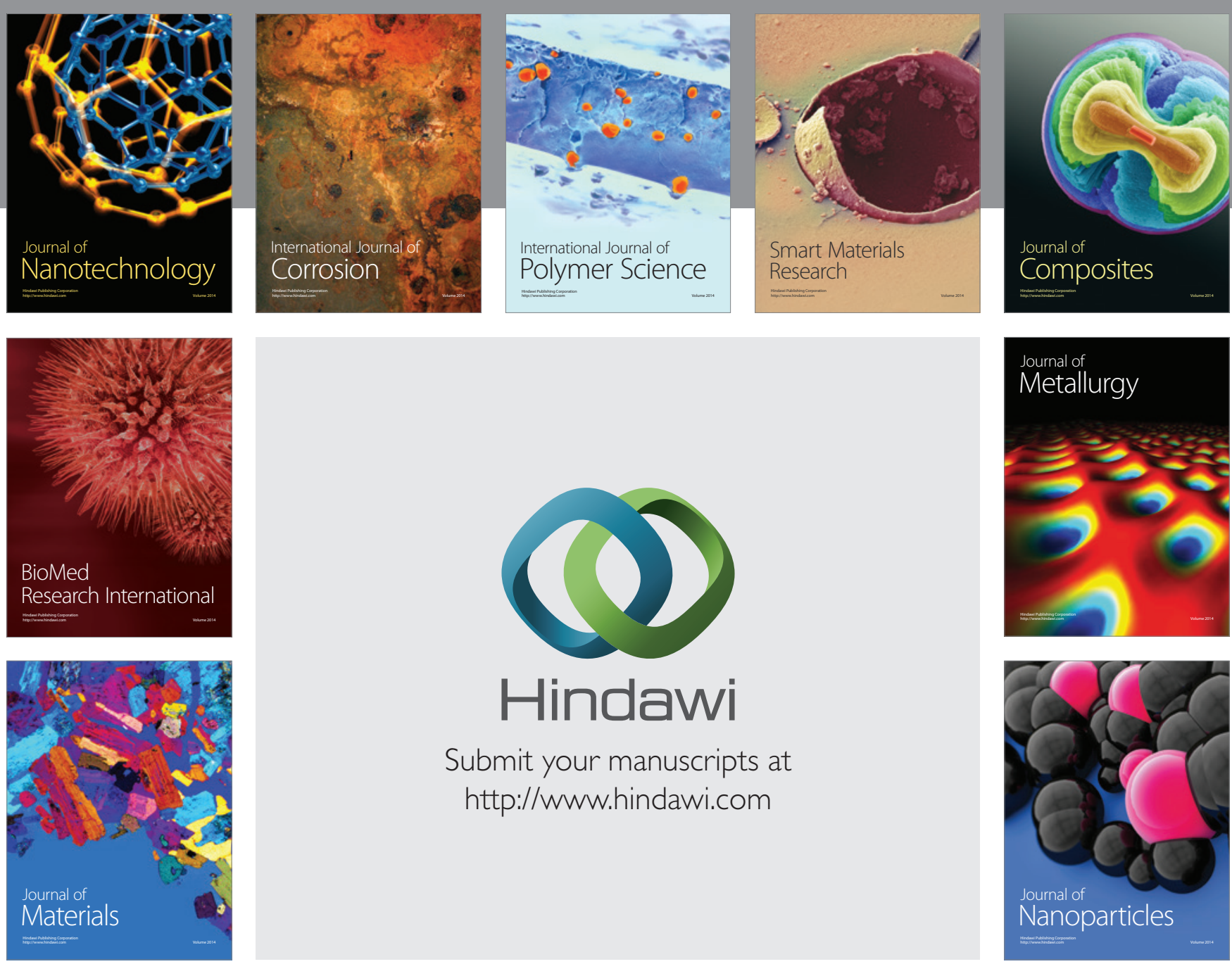

Submit your manuscripts at http://www.hindawi.com
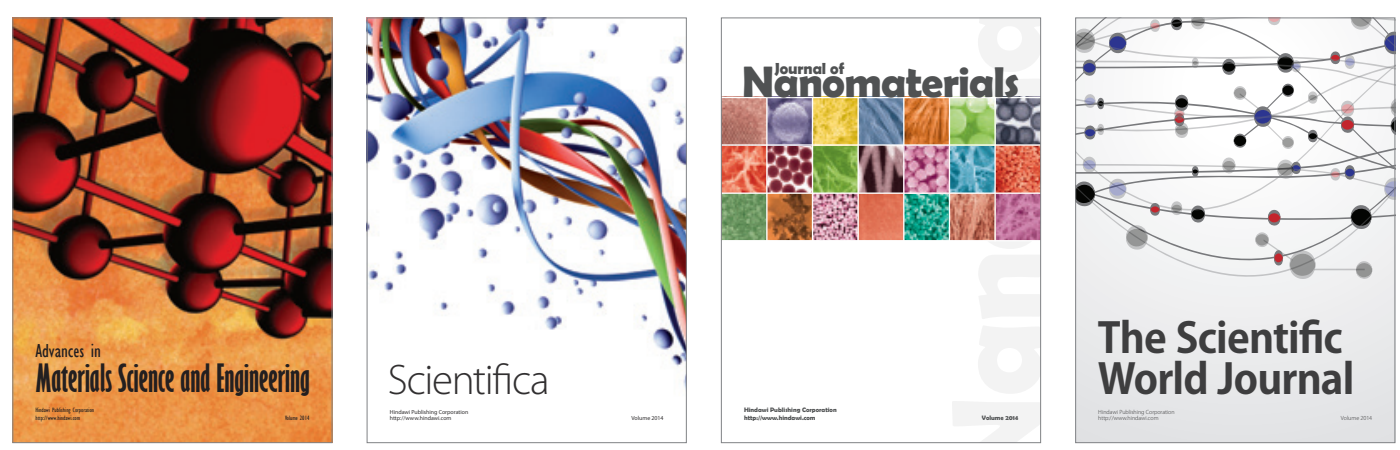

\section{The Scientific World Journal}
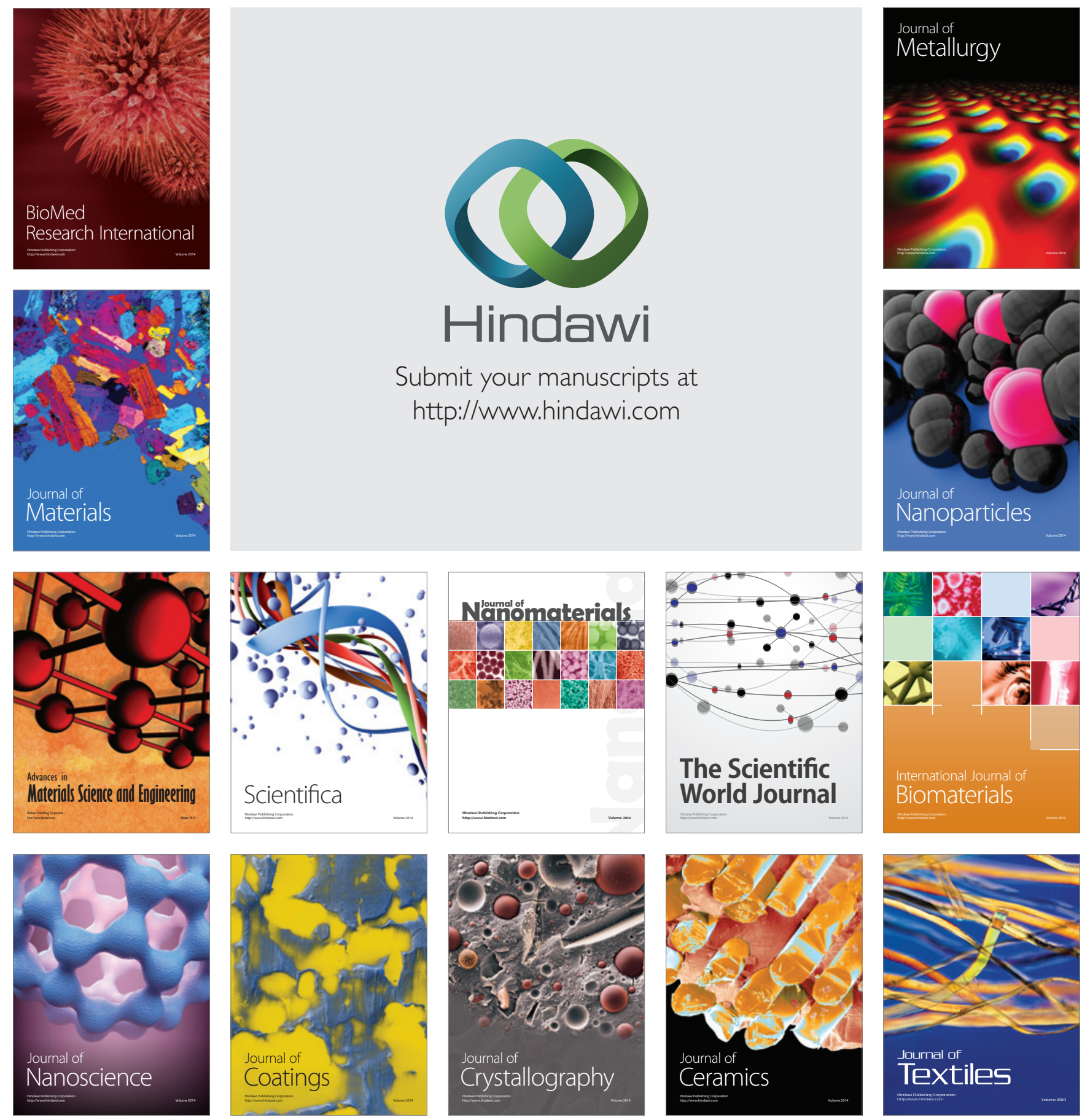